\title{
Lade and Modified Lade 3D Rock Strength Criteria
}

\author{
Sergio A. B. da Fontoura
}

Published online: 18 July 2012

(C) Springer-Verlag 2012

\section{List of Symbols}

$a, m, \eta_{1} \quad$ Parameters of original Lade formulation

c Cohesion

$C_{0} \quad$ Uniaxial compressive strength

$I_{1}^{\prime} \quad$ First invariant of the effective stress tensor

$I_{3}^{\prime} \quad$ Third invariant of the effective stress tensor

$I_{1}^{\prime \prime} \quad$ Modified first invariant of effective stresses

tensor in modified Lade

$I_{3}^{\prime \prime} \quad$ Modified third invariant of effective stresses tensor in modified Lade

$p_{\mathrm{a}} \quad$ Atmospheric pressure

$P_{\mathrm{p}} \quad$ Pore fluid pressure

$S_{\mathrm{a}}, \eta \quad$ Parameters of modified Lade formulation

$T_{0} \quad$ Uniaxial tensile strength

$\alpha \quad$ Biot's parameter

$\phi \quad$ Angle of internal friction

$\sigma_{1}^{\prime} \quad$ Major principal effective stress

$\sigma_{2}^{\prime} \quad$ Intermediate principal effective stress

$\sigma_{3}^{\prime} \quad$ Minor principal effective stress

\section{Description}

This part describes the failure criterion known as Lade criterion (LC) and its variation, the so-called modified Lade criterion (MLC) as proposed by Ewy (1999). The two failure criteria are described first, including the material parameters involved. Next is shown how to determine these

S. A. B. da Fontoura ( $\square)$

Department of Civil Engineering, Pontifical Catholic University of Rio de Janeiro, Rio de Janeiro, Brazil

e-mail: fontoura@puc-rio.br parameters from laboratory experiments conducted on rock samples. Then follows a section on the validation of each criterion. In the same section, there is a discussion on how to evaluate the stresses at failure knowing the material parameters. Advantages and disadvantages of these two criteria are highlighted at the end of this report, followed by suggestions of when these two failure criteria should be applied for evaluating rock strength.

\section{Background}

The strength of isotropic rocks is a function of the three principal effective stresses acting upon the material and may be represented by a surface in the principal stress space, as described by Eq. (1), and the indexes 1, 2 and 3 refer to the principal directions of the stress field. For porous, saturated rocks, the effective stresses follow the definition by Terzaghi and later modified by Biot, Eq. (2), where $\alpha$ is the so-called Biot's parameter, and $P_{\mathrm{p}}$ is the pore fluid pressure, and $\sigma$ is the total stress.

$f\left(\sigma_{1}^{\prime}, \sigma_{2}^{\prime}, \sigma_{3}^{\prime}\right)=0$

$\sigma^{\prime}=\sigma-\alpha P_{\mathrm{p}}$

Figure 1 shows the results of stresses at failure obtained from different tests and plotted on the octahedral plane characterized by a given value of the first stress invariant, $I_{1}$. The curve displayed and adjusted to the test results represents the intersection between the failure surface and the octahedral plane.

It is common practice in geotechnical engineering to use a particular form of Eq. (1) such as Mohr-Coulomb and Hoek-Brown failure criteria, that do not recognize the importance of the intermediate principal effective stress, $\sigma_{2}^{\prime}$, on the ultimate rock failure load. However, small-scale 


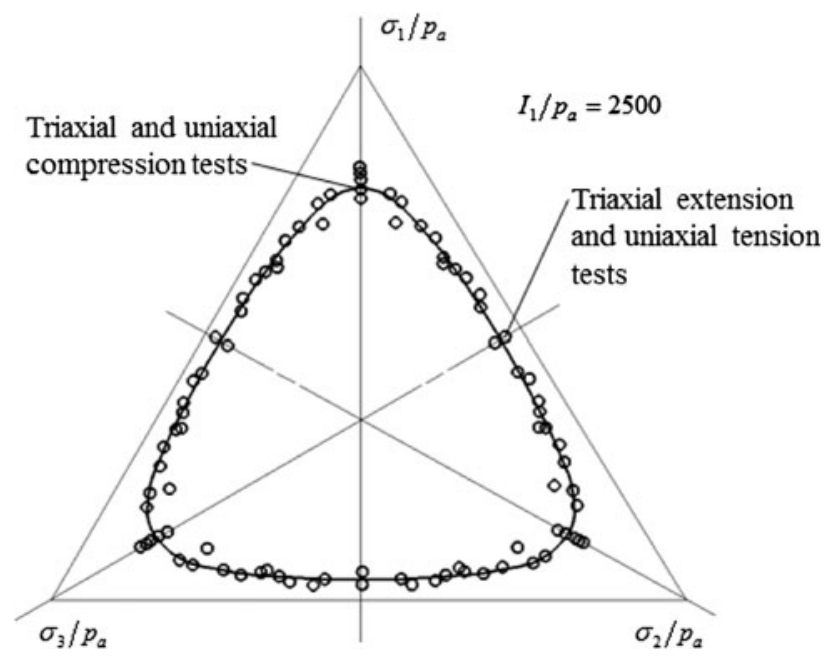

Fig. 1 Results of triaxial, biaxial and cubical triaxial compression tests on sandstone by Akai and Mori (1970) displayed on a given octahedral plane (Lade 1993)

laboratory experiments have proven that rock strength depends on all three principal stresses and several failure criteria have been developed to describe such a dependency. Lade (1993) presents a review of several 3D failure criteria for evaluating strength of materials, in particular of soils and rocks, and concludes that none of these criteria represent, with reasonable accuracy, the $3 \mathrm{D}$ nature of rock failure.

Lade's failure criterion was initially developed for soils which present very low to no cohesion, Lade (1977), and later on modified to include materials such as concrete and rocks (Lade 1982; Kim and Lade 1984). Differently from other 3D failure criteria that relate the first stress invariant with the second deviatoric stress invariant, Lade's failure criterion uses a special relationship between the first and the third stress invariants. Ewy (1999) proposed a failure criterion based upon Lade's criterion, and named modified Lade criterion, which is forced to coincide with the MohrCoulomb failure criterion under the conditions of triaxial compression test, i.e., $\sigma_{1}>\sigma_{2}=\sigma_{3}$. Ewy's motivation was to develop a simple methodology to take into account the effect of the intermediate principal stress on wellbore stability calculations.

\section{Formulation, Parameters and Validation}

\subsection{Original Lade Criterion}

\subsubsection{Formulation}

The original form of Lade's criterion for rocks is expressed as:
$\left(\frac{I_{1}^{\prime 3}}{I_{3}^{\prime}}-27\right)\left(\frac{I_{1}^{\prime}}{p_{\mathrm{a}}}\right)^{m}=\eta_{1}$

In Eq. (3), $I_{1}^{\prime}$ and $I_{3}^{\prime}$ are, respectively, the first and the third invariants of the effective stress tensor at failure, modified by a translation of axes of $a p_{\mathrm{a}}$ in the space of principal stresses along its hydrostatic axis in order to accommodate tensile strength, see Fig. 2, as discussed by Kim and Lade (1984). Equations (4) and (5) describe the stress invariants and the stresses are defined in Eq. (6). In these equations, $p_{\mathrm{a}}$ is the atmospheric pressure in the same unit system as the stresses. For instance, if the stresses are expressed in $\mathrm{MPa}, p_{\mathrm{a}}$ is $10^{-1} \mathrm{MPa}$.

$I_{1}^{\prime}=\bar{\sigma}_{1}+\bar{\sigma}_{2}+\bar{\sigma}_{3}$

$I_{3}^{\prime}=\bar{\sigma}_{1} \cdot \bar{\sigma}_{2} \cdot \bar{\sigma}_{3}$

$\bar{\sigma}_{1}=\sigma_{1}^{\prime}+a p_{\mathrm{a}} ; \quad \bar{\sigma}_{2}=\sigma_{2}^{\prime}+a p_{\mathrm{a}} ; \quad \bar{\sigma}_{3}=\sigma_{3}^{\prime}+a p_{\mathrm{a}}$

For intact rocks there are three parameters to be determined in order to describe the 3D nature of the failure criterion: $a, m$ and $\eta_{1}$. The evaluation of these parameters is explained next. As should be expected, these three parameters, $a, m$ and $\eta_{1}$, influence the shape and location of the failure surface as discussed by Kim and Lade (1984).

\subsubsection{Evaluating Rock Parameters from Experiments}

Lade failure criterion describes failure of rocks subjected to three different effective principal stresses, even though the most common test is the so-called compression, axisymmetric triaxial test, where $\sigma_{1}^{\prime}>\sigma_{2}^{\prime}=\sigma_{3}^{\prime}$. True triaxial tests

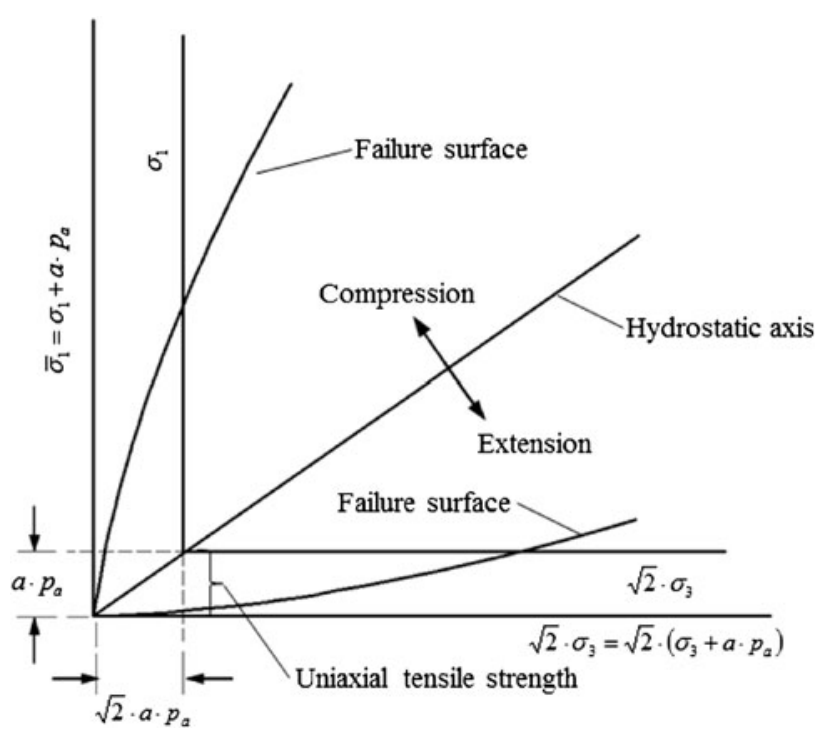

Fig. 2 Translation of principal stress space along hydrostatic axis to include effect of tensile strength in failure criterion (Lade 1993) 
are less common $\left(\sigma_{1}^{\prime}>\sigma_{2}^{\prime}>\sigma_{3}^{\prime}\right)$ but can also be interpreted through this failure criterion.

Parameter $a$ The value of $a$ can be estimated by considering that $a p_{\mathrm{a}}$ is equal or very close to the tensile strength of the rock, $T_{0}$. If the tensile strength is not determined directly or indirectly through the Brazilian tensile test, the empirical formulation, proposed by Lade (1993), Eq. (7), can be used, where $C_{0}$ is the unconfined compressive strength and $T$ and $t$ are material parameters. Lade (1993) suggests values of $T$ and $t$ as a function of rock type based on a large set of tests. Average values for $T$ and $t$, are, respectively, -0.219 and 0.825 .

$T_{0}=T p_{\mathrm{a}}\left(\frac{C_{0}}{p_{\mathrm{a}}}\right)^{t}$

Parameters $m$ and $\eta_{1}$ Using the value of $a$ determined as explained above, each data point corresponding to failure, and obtained experimentally, can generate a pair of values of $A$ and $B$, Eqs. (8) and (9). These two numbers, plotted in a log-log diagram, generate a theoretical linear relationship, Eq. (10), and the parameters $m$ and $\eta_{1}$ can be obtained by linear regression.

$A=\left(\frac{I_{1}^{\prime 3}}{I_{3}^{\prime}}\right)-27$

$B=\left(\frac{p_{\mathrm{a}}}{I_{1}^{\prime}}\right)$

In a $\log -\log$ space, Eq. (3) may be expressed as Eq. (10).

$\log A=m \log B+\log \eta_{1}$

The parameters $m$ and $\eta_{1}$ can be obtained by least-square fitting Eq. (10) through the experimental data. Figure 3 indicates a typical example.

\subsubsection{Validation of the Lade Failure Criterion: Applications}

Lade (1993) describes the interpretation of about 90 data sets obtained from the literature and covering different types of igneous, metamorphic and sedimentary rocks. Different types of tests were used to obtain the data including the simple ones such as unconfined compression test, uniaxial tension test, triaxial compression test and the less common ones such as triaxial extension test, biaxial compression test, cubical triaxial test, and torsion shear test on hollow cylinder with axial loading. Two types of tests were considered to be unreliable for the purpose of obtaining data set for interpretation: internal and external pressure on hollow cylinders combined with axial loading and torsional tests on solid samples. The validation of the failure criterion proposed was acceptable and, as it should be expected, the range of values of the parameters was very

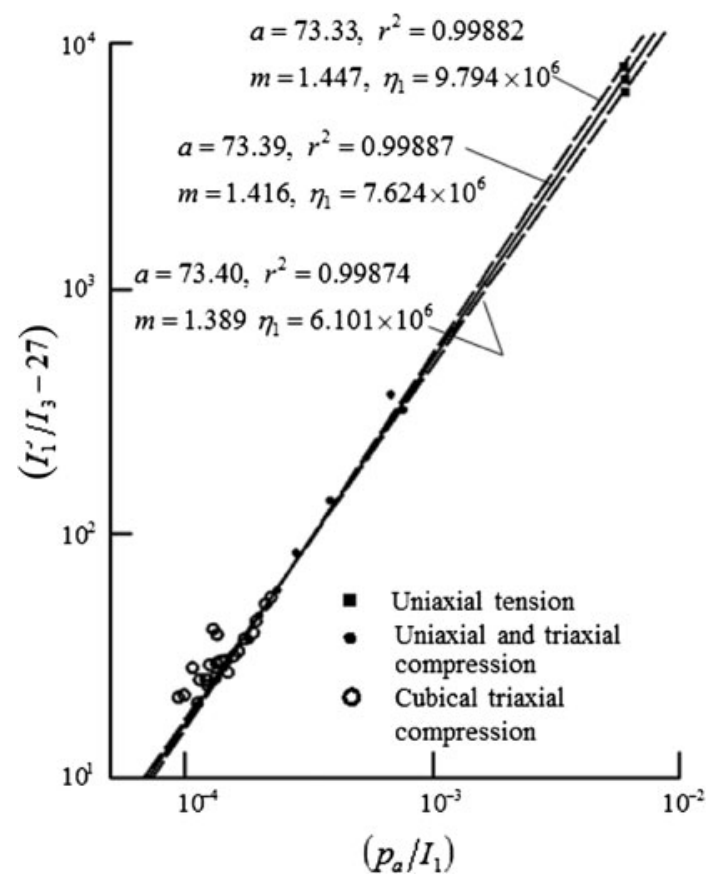

Fig. 3 Determination of material parameters involved in the failure criterion for Mizuho Trachyte tested by Mogi (1971) and (Lade 1993)

wide. Only in few cases the regression coefficient was low in the range of 0.1-0.40 mainly in coals, suggesting that anisotropy may play a role in the application and validation of the failure criterion.

\subsubsection{Evaluating Stresses at Failure}

An important issue is the evaluation of the stress at failure knowing the material parameters and two principal stresses at failure. This is the operation to be carried out when plotting the failure surface knowing the material parameters, i.e., for each pair of values of two principal stresses, the third one must be determined (Fig. 4). Equation (3) must be solved but it does not end up being a simple matter since the final equation is of the transcendental type and must be solved numerically or graphically.

\subsection{Modified Lade Criterion}

\subsubsection{Formulation}

Ewy (1999) presented a simplified version of the Lade criterion that can be expressed by Eq. (11). Comparing Eqs. (11) with (3) one can observe both the similarity and the difference: the non-linear dependence upon $I_{1}$ to the power $m$ was removed which is equivalent to make $m$ equal to zero. Another important difference between the two criteria is the translation of axis. The term $S_{\text {a }}$ proposed by Ewy 
Fig. 4 Comparison of failure criterion with results of tests on sandstone performed by Akai and Mori (1970) in a triaxial plane, $\mathbf{b}$ biaxial plane and c octahedral plane (Lade 1993)

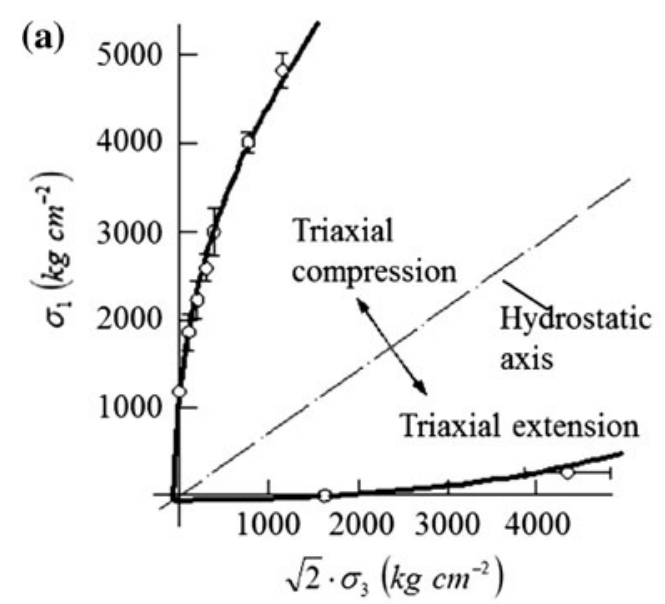

(b)

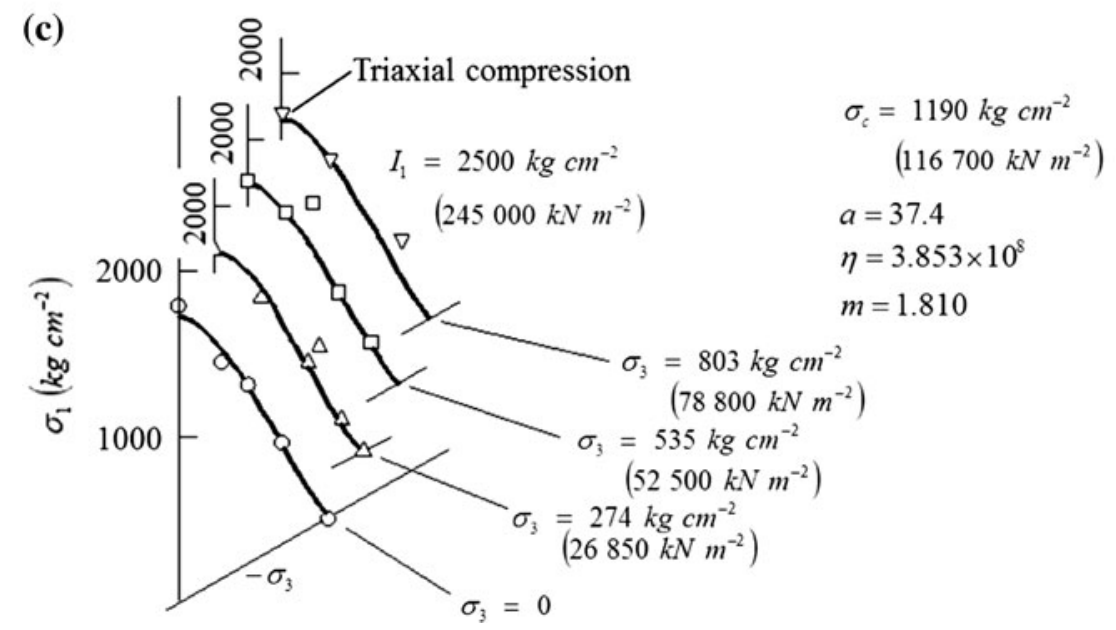

(1999) is related to both the cohesion and friction angle as defined by the Mohr-Coulomb failure criterion.

$$
\begin{aligned}
& \left(\frac{\left(I_{1}^{\prime \prime}\right)^{3}}{I_{3}^{\prime \prime}}\right)=27+\eta \\
& I_{1}^{\prime \prime}=\left(\sigma_{1}+S_{\mathrm{a}}-P_{\mathrm{p}}\right)+\left(\sigma_{2}+S_{\mathrm{a}}-P_{\mathrm{p}}\right)+\left(\sigma_{3}+S_{\mathrm{a}}-P_{\mathrm{p}}\right)
\end{aligned}
$$

$$
I_{3}^{\prime \prime}=\left(\sigma_{1}+S_{\mathrm{a}}-P_{\mathrm{p}}\right)\left(\sigma_{2}+S_{\mathrm{a}}-P_{\mathrm{p}}\right)\left(\sigma_{3}+S_{\mathrm{a}}-P_{\mathrm{p}}\right)
$$

\subsubsection{Material Parameters}

As presented in Eqs. (11) and (12a, b), the MLC requires two parameters to describe the rock strength: $S_{\mathrm{a}}$ and $\eta$. The parameter $S_{\mathrm{a}}$, that represents the axis translation, is made equal to $c / \tan \phi$, ( $c$ is the cohesion and $\phi$ is the friction angle in the Mohr-Coulomb failure criterion), which is equivalent to translate the $\tau$ axis, in the $\sigma-\tau$ space, in order to make a cohesive material appear like a cohesionless one. In order to obtain the material parameter $\eta$, as described by Eq. (14), the MLC is forced to coincide with the MohrCoulomb failure criterion when the stress state coincides with the triaxial compression test $\left(\sigma_{1}>\sigma_{2}=\sigma_{3}\right)$, and that means that the MLC is linear in the space $\sigma_{3}-\sigma_{1}$ (or $\sqrt{ } 2 \sigma_{2}-\sigma_{1}$ or $p-q$ ).

$S_{a}=\frac{c}{\tan \phi}$

$\eta=4 \cdot(\tan \phi)^{2}\left\{\frac{9-7 \sin \phi}{1-\sin \phi}\right\}$

\subsubsection{Validation of MLC: Applications}

Ewy (1999) presents some numerical experiments generating strength envelopes using the MLC for a given set of $c$ and $\phi$ and demonstrates that the results are similar, in trend, to experimental results of polyaxial tests described in the literature. Several investigators have tested the MLC in their experiments and concluded that the criterion represented well the test results. Colmenares and Zoback (2002) investigated the application of MLC to five different types of rocks, all of high strength and of very brittle nature (Dunham dolomite: $C_{0}=450 \mathrm{MPa}$ and $\phi=33^{\circ}$, Shivahama sandstone: $C_{0}=95 \mathrm{MPa}$ and $\phi=38.6^{\circ}$, Yuubari 

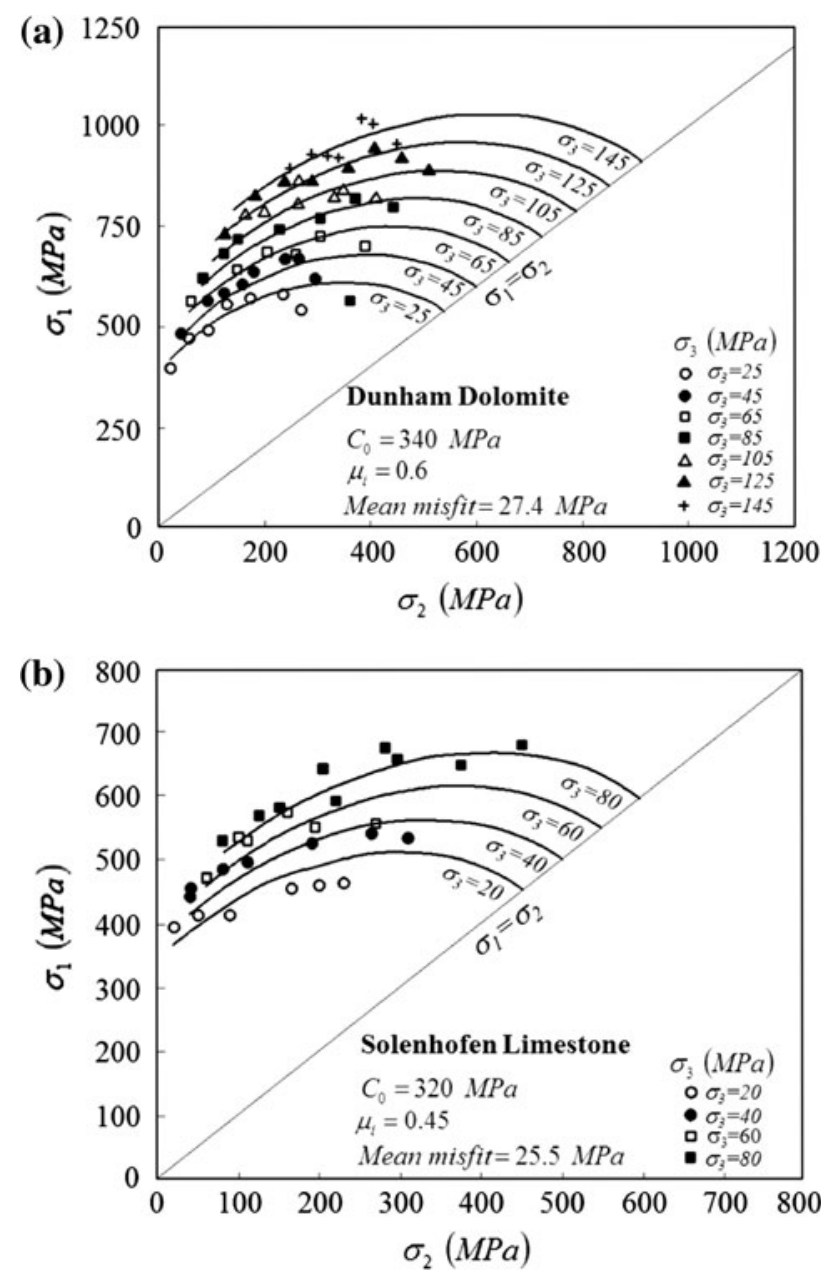

Fig. 5 Fitting modified Lade criterion through polyaxial test carried out on a Dunham dolomite and b Solnhofen limestone (Colmenares and Zoback 2002)

shale: $C_{0}=120 \mathrm{MPa}$ and $\phi=26.5^{\circ}$, Solenhofen limestone: $C_{0}=375 \mathrm{MPa}$ and $\phi=28.8^{\circ}$, KTB amphibolite: $C_{0}=300 \mathrm{MPa}$ and $\phi=50.1^{\circ}$ ). Figure 5 displays the use of MLC to the experimental data from Dunham dolomite and Solnhofen limestone suggesting a very good fit when minimizing the mean standard deviation misfit to the test results.

\subsubsection{Evaluating Stresses at Failure}

An important issue is the evaluation of the stress at failure knowing the material parameters and two principal stresses at failure. This is the operation to be carried out when plotting the failure surface knowing the material parameters. For each pair of values of principal stresses, the third one must be determined. Equation (11) is to be solved and that leads to a 3rd degree equation in the unknown stress that has to be obtained numerically and the appropriate solution, of the three possible ones, selected.

\section{Advantages and Disadvantages}

The 3D strength criteria, LC and MLC, proposed do note generate sharp corners in the failure surface allowing the continuity of the first derivative with respect to the stresses. The LC requires the determination of three parameters but imposes no restrictions to the experimental data neither assumes any special shape of the strength envelope. There are indications that the criterion suits well a very large range of rocks tested under different stress conditions as presented by Lade (1993). Very important, the parameters can be determined using data set obtained from very simple unconfined compression tests and triaxial compression tests. An important limitation of LC is that the parameters associated with this criterion do not have a clear relationship with the most commonly used $c$ and $\phi$ from the Mohr-Coulomb failure criterion. This somehow limits the application of Lade's criterion in practice.

The MLC was devised with the possibility of application in mind and therefore has components that make the method attractive. The parameters can be determined as a function of the well known $c$ and $\phi$ from the Mohr-Coulomb failure which increases its attractiveness for applications in the oil industry since there are hardly any samples available for experiments and the shear strength parameters are obtained through correlations with well logs. Implicit in the MLC is the linear nature of the shear strength envelope when interpreting the results of compression triaxial test. This implies that the MLC cannot handle experimental data from rocks that behave in a non-linear manner, which is the case of high pressure, high temperature applications. The LC does not offer this restriction.

\section{Recommendations}

The use of both criteria is restricted to cases where the intact rock behavior is representative of the problem. The wellbore behavior problem in the oil industry is a natural candidate for the application of both LC and MLC. Ewy (1999) and Yi et al. (2006) have reported the application of MLC to investigate the effect of the intermediate stress in the wellbore stability and Yi et al. (2005) have also used the MLC to study the onset of sand production around producing wells.

\section{References}

Akai K, Mori H (1970) Ein versuch uber Bruchmecanismus von Sandstein under mehrachsigen Spannungzustand. In: ISRM Proc. 2nd Int Congress Rock Mech. ISRM, Belgrad, vol 2, Paper 3-30

Colmenares LB, Zoback MD (2002) A statistical evaluation of intact failure criteria constrained by polyaxial test data for five 
different rocks. Int J Rock Mech Min Sci Geom Abstr 29:695-729

Ewy RT (1999) Wellbore stability prediction by use of a modified Lade criterion. SPE Drill Complet 14:85-91

Kim MK, Lade PV (1984) Modeling rock strength in three dimensions. Int J Rock Mech Min Sci Geom Abstr 21:21-33

Lade PV (1977) Elasto-plastic stress-strain theory for cohesionless soils with curved yield surfaces. Int J Solids Struct 13:10191035

Lade PV (1982) Three-parameter failure criterion for concrete. J Eng Mech DivAm Soc Civ Eng 108:850-863
Lade PV (1993) Rock strength criteria: the theories and the evidence. In: Hudson J, Brown ET (eds) Comprehensive rock engineering. Elsevier Inc, London, pp 225-284

Mogi K (1971) Fracture and flow of rocks under high triaxial compression. J Geophys Res 76:1255-1269

Yi X, Valkó PP, Russell JE (2005) Effect of rock strength criterion on the predicted onset of sand production. Int J Geomech ASCE 5(1):66-73

Yi X, Ong S, Russell JE (2006) Quantifying the effect of rock strength criteria on minimum drilling mud weight prediction using polyaxial rock strength test data. Int J Geomech ASCE 6(4):260-268 